\title{
Facharztprüfung Innere Medizin Begleiterkrankungen bei Hypertonie
}

? Sie betreuen eine 54-jährige Patientin mit einem neu aufgetretenen Hypertonus. Die Patientin, die früher als sehr aktiv galt, klagt über Muskelschwäche, Müdigkeit und Antriebslosigkeit. Der körperliche Untersuchungsbefund ist unauffällig. In der Laboruntersuchung fällt ein erniedrigtes Kalium von $3,0 \mathrm{mmol} / 1$ auf. Woran denken Sie?

Antwort An einen hypokaliämischen Hypertonus.

Kommentar Hypokaliämischer Hypertonus:

- V.a. sekundären Hypertonus,

- Notwendigkeit einer speziellen Abklärung.

? Was meinen Sie mit „spezieller Abklärung“?

Antwort Abklärung der Differenzialdiagnosen: insbesondere Medikamenteneinnahme, die zur Hypokaliämie führt, aber auch das Conn-Syndrom.

Kommentar Differenzialdiagnose des hypokaliämischen Hypertonus:

- essenzielle Hypertonie und Diuretikaeinnahme: sekundärer Hyperaldosteronismus bei renalem Natriumentzug,

- primärer Hyperaldosteronismus: Conn-Syndrom,

- renovaskulärer Hypertonus mit sekundärem Hypoaldosteronismus,

- selten andere Ursachen.

? Wie gehen Sie in dieser Situation weiter vor?

Antwort Wichtigste Maßnahme ist die Medikamentenanamnese, da die medikamentöse Behandlung eines Hypertonus die häufigste Ursache der Hypokaliämie ist. Ggf. sollten die Medikamente abgesetzt und der Kaliumspiegel kontrolliert werden.
Kommentar Medikamente, die bei hypokaliämischem Hypertonus berücksichtigt werden sollten:

- Diuretika,

- Laxanzien,

- Kortikosteroide.

? Angenommen, die Patientin nimmt gar keine Medikamente, weder Blutdruckmedikamente noch Laxanzien. Was machen Sie dann?

Antwort In diesem Falle muss ein pathologischer Hyperaldosteronismus ausgeschlossen werden.

Kommentar Aldosteronwirkung: Natriumretention mit Hypervolämie, Kaliumexkretion.

? An welche Ursachen eines pathologischen Hyperaldosteronismus denken Sie?

Antwort An einen primären Hyperaldosteronismus, das Conn-Syndrom, aber auch an einen sekundären Hyperaldosteronismus bei renovaskulärem und renoparenchymatösem Hochdruck.

Kommentar Differenzialdiagnose des Hyperaldosteronismus:

- primärer Hyperaldosteronismus,

- Stimulation des Renin-AldosteronAngiotensin-Systems: Nierenarterienstenose, maligne Hypertonie, Reninom (selten).

? Sie haben den Verdacht, dass Ihnen die Patientin einen Laxanzienabusus verschweigt. Gibt es eine einfache Untersuchung, dies herauszubekommen?

Antwort Ja. Die Bestimmung der Kaliumausscheidung im Urin.

Kommentar Hypokaliämie:

- Kaliumausscheidung im Urin $<30 \mathrm{mmol} / \mathrm{d}$ : V.a. Laxanzienabusus,

- Kaliumausscheidung im Urin $>30 \mathrm{mmol} / \mathrm{d}$ : V.a. Hyperaldosteronismus.
7 Sie haben einen Laxanzienabusus ausgeschlossen, die Kaliumausscheidung ist hoch. Was machen Sie jetzt?

Antwort Bestimmung von Aldosteron und Renin im Serum.

Kommentar Der Nachweis eines Hyperaldosteronismus und die Differenzierung zwischen einer primären und einer sekundären Form ist möglich anhand der Bestimmung von Aldosteron und Renin im Serum.

? Bei der Patientin liegt eine deutliche Erhöhung des Aldosterons und eine Erniedrigung des Renins vor. Worum wird es sich handeln?

Antwort Um ein Conn-Syndrom, den primären Hyperaldosteronismus.

Kommentar Hypokaliämische Hypertonie, Aldosteron hoch, Renin niedrig:

- primärer Hyperaldosteronismus

(Conn-Syndrom),

- andere Ursachen sind sehr selten.

? Wie kommt es überhaupt zu einem primären Hyperaldosteronismus?

Antwort Durch ein Adenom oder eine bilaterale Hyperplasie der Nebennieren.

Kommentar Primärer Hyperaldosteronismus:

- Adenom: $70 \%$,

- bilaterale Hyperplasie: $30 \%$,

- Karzinom: Rarität.

? Sie haben jetzt einen primären Hyperaldosteronismus nachgewiesen. Wie gehen Sie weiter vor?

Antwort Lokalisationsdiagnostik mittels Sonografie, CT und MRT. 
? Sie behandeln eine 62-jährige Patientin mit Hypertonus. Bei ihr bestehen eine ausgeprägte Stammfettsucht, ein Hirsutismus sowie auffällig schlanke Extremitäten. Woran denken Sie?

Antwort An ein Cushing-Syndrom.

Kommentar Hypertonus plus Adipositas: immer an Cushing-Syndrom denken.

Wie gehen Sie vor?

Antwort Nachweis bzw. Auschluss des Hyperkortisolismus durch Bestimmung des freien Kortisols im 24-Stunden-Urin. Durchführung eines Kortisol-Tagesprofils.

Kommentar Nachweis eines Hyperkortisolismus:

1. freies Kortisol im 24-Stunden-Urin,

2. Kortisol-Tagesprofil:

- Nachweis der aufgehobenen Tagesrhythmik,

3. Dexamethason-Hemmtest:

- Gabe von Dexamethason 2 mg p.o. abends,

- Bestimmung des morgendlichen Plasma-Kortisols,

- ein Wert > $5 \mathrm{mg} / \mathrm{dl}$ spricht für ein Cushing-Syndrom.

? Angenommen, der DexamethasonHemmtest und das Kortisol im Urin sind unauffälig. Ist damit ein CushingSyndrom ausgeschlossen?

Antwort Ja.

Kommentar Ein normaler Dexamethason-Hemmtest und eine normale Kortisolausscheidung im Urin schließen einen Hyperkortisolismus aus.
? Nehmen wir jetzt aber an, Sie haben bei der Patientin einen Hyperkortisolismus nachgewiesen. Wie geht es jetzt weiter?

Antwort Es sollte zunächst eine ätiologische Zuordnung erfolgen.

Kommentar Reihenfolge der Diagnostik bei Hypertonus und V.a. Hyperkortisolismus:

1. Nachweis des Hyperkortisolismus,

2. Nachweis der Ätiologie,

3. Lokalisationsdiagnostik.

? Was meinen Sie mit Nachweis der Ätiologie?

Antwort Der Hyperkortisolismus kann viele Ursachen haben. Häufigste Ursache ist die exogene Zufuhr von Kortikosteroiden. Dagegen sind endogene ACTHabhängige (Morbus Cushing) oder ACTHunabhängige Formen selten.

Kommentar Ätiologie des CushingSyndroms:

exogen:

- Therapie mit Kortikosteroiden (häufigste Form), endogen ACTH-abhängig (85\% der endogenen Formen):

- Hypophysenadenome (=zentrales Cushing-Syndrom = Morbus Cushing, $80 \%$ der ACTH-abhängigen Formen),

- ektope ACTH-Produktion (= paraneoplastisch, 20\% der ACTH-abhängigen Formen), endogen ACTH-unabhängig (15\% der endogenen Formen, adrenales Cushing-Syndrom):

- Nebennierenadenome,

- Nebennierenkarzinome,

- Nebennierenhyperplasie (bilateral).
Wie differenzieren Sie diese Formen?

Antwort Zunächst Bestimmung von ACTH im Plasma. Beim adrenalen Cushing-Syndrom ist es erniedrigt, beim zentralen Cushing-Syndrom ist es normal oder erhöht. Bei der ektopen ACTHProduktion ist ACTH immer erhöht oder deutlich erhöht.

Kommentar ACTH beim CushingSyndrom: adrenales Cushing-Syndrom:

- ACTH niedrig, zentrales Cushing-Syndrom:

- ACTH normal oder erhöht, paraneoplastisches Cushing-Syndrom:

- ACTH erhöht oder stark erhöht.

2 Kennen Sie die Grunderkrankungen bei paraneoplastischer ACTH-Produktion?

Antwort Bronchialkarzinome, Ovarialkarzinome, gastrointestinale Karzinome, Karzinoide.

Kommentar Paraneoplastische ACTHProduktion:

- kleinzelliges Bronchialkarzinom (häufigste Ursache),

- Ovarialkarzinom (gastrointestinale Karzinome),

- Karzinoide.

Regel Die häufigste Ursache einer paraneoplastischen ACTH-Produktion ist das kleinzellige Bronchialkarzinom.
Nach: Berthold Block, Facharztprüfung Innere Medizin, 3000 kommentierte Prüfungsfragen

4. Aufl., kompl. überarb. akt. 2011, 576 S., 106 Abb., kart. ISBN: 9783131359544 